\title{
The pro-apoptotic effects of TIPE2 on AA rat fibroblast-like synoviocytes via regulation of the DR5-caspase-NF-kB pathway in vitro
}

This article was published in the following Dove Press journal:

OncoTargets and Therapy

29 February 2016

Number of times this article has been viewed

\author{
Chunyan Shi',2,* \\ Shifeng Zhang ${ }^{1,3, *}$ \\ Shifu Hong' \\ Jinglong Pang' \\ Yeletai Yesibulati' \\ Ping Yin ${ }^{4}$ \\ Guohong Zhuang' \\ 'Organ Transplantation Institute, Anti- \\ Cancer Research Center, Medical \\ College, Xiamen University, Xiamen, \\ Fujian, ${ }^{2}$ The Department of Oncology, \\ Jiujiang No.I People's Hospital, \\ Jiujiang, Jiangxi City, ${ }^{3}$ Division of \\ Gastroenterology Surgery, Zhongshan \\ Hospital, Gastroenterology \\ Institute of Xiamen University, \\ Gastroenterology Center of Xiamen, \\ ${ }^{4}$ The Department of Pathology, \\ Zhongshan Hospital, Xiamen \\ University, Xiamen, Fujian, People's \\ Republic of China \\ *These authors contributed equally \\ to this work
}

Correspondence: Ping Yin

The Department of Pathology, Zhongshan Hospital, Xiamen University, Hubin South Road, Xiamen 36II00, Fujian, People's Republic of China Email yinping2002@163.com

Guohong Zhuang

Organ Transplantation Institute, Anti-Cancer Research Center, Medical College, Xiamen University, Xiang'an South Road, Xiamen 36II00, Fujian,

People's Republic of China

Tel +865922187157

Fax +86 5922187157

Email zghxmu@163.com
Abstract: TIPE2, also known as TNFAIP8L2, a member of the tumor necrosis factoralpha-induced protein-8 (TNFAIP8) family, is known as an inhibitor in inflammation and cancer, and its overexpression induces cell death. We examined the role of TIPE2 with respect to adjuvant arthritis (AA)-associated pathogenesis by analyzing the TIPE2 regulation of death receptor (DR5)-mediated apoptosis in vitro. The results showed that TIPE2 was detected in normal fibroblast-like synoviocytes (FLSs), but scarcely observed in AA-FLSs. Therefore, recombinant MIGR1/TIPE2 ${ }^{+/+}$and control MIGR1 lentivirus vectors were transfected to AA-FLSs, which were denoted as TIPE2 ${ }^{+/+}$-FLSs and MIGR1-FLSs, respectively. Our results showed that TIPE2 ${ }^{+++}$-FLSs were highly susceptible to ZF1-mediated apoptosis, and ZF1 was our own purification of an anti-DR5 single chain variable fragment antibody. Under the presence of TIPE2, the expression of DR5 was significantly increased compared with that of the

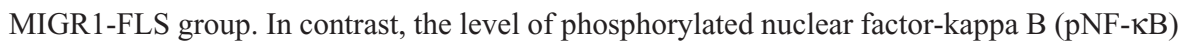
was lower in the TIPE2 ${ }^{+/+}$-FLS group treated with ZF1, whereas the activity of caspase was higher. Moreover, the rate of apoptosis in the TIPE2 ${ }^{+/+}$-FLS group, which was pretreated with caspase inhibitor Z-VAD-FMK, was significantly decreased. In contrast, the apoptosis occurrence in the MIGR1-FLS group increased significantly with the pretreatment of the NF- $\kappa B$ inhibitor Bay. These results indicated that TIPE2 increased the apoptosis of AA-FLSs by enhancing DR5 expression levels, thereby promoting the activation of caspase and inhibiting the activation of NF-KB in AA-FLSs. TIPE2 might potentially act as a therapeutic target for rheumatoid arthritis.

Keywords: rheumatoid arthritis, adjuvant arthritis, fibroblast-like synoviocytes, DR5, TIPE2

\section{Introduction}

Rheumatoid arthritis (RA) synovial tissue contains large numbers of infiltrated inflammatory cells and excessively proliferated synovial lining cells. ${ }^{1}$ Fibroblastlike synoviocytes (FLSs), which fail to precede apoptosis, play an important role in RA pathology. ${ }^{2-4}$ Adjuvant arthritis (AA) is a model of experimental arthritis that is induced by the injection of complete Freund's adjuvant. ${ }^{5}$ The symptoms of AA in joints, along with the cellular and humoral immunities, show a similar pathology to those observed in RA.

Tumor necrosis factor (TNF)-related apoptosis-inducing ligand (TRAIL) induces apoptosis by binding to TRAIL receptor 1 (death receptor 4, DR4) and TRAIL receptor 2 (DR5), which antagonize the death signal. ${ }^{6}$ Previous reports have indicated that RA synovial tissue and fibroblasts express high levels of DR5 and are highly susceptible to DR5-mediated apoptosis. ${ }^{7}$ A novel agonistic antihuman DR5 single chain variable 
fragment antibody (Ab), $\mathrm{ZF} 1$, is known to induce strong apoptosis response without hepatocellular toxicity. ${ }^{8}$ Although the high expression levels of DR5 in RA synovial fibroblasts are thought to sensitize these cells for apoptosis, cell hyperplasia and lymphocyte activation are severe.

A new member of the TNF-alpha-induced protein- 8 (TNFAIP8) family, which is commonly referred to as the TIPE family, was recently identified from an inflamed spinal cord, this is known as TNFAIP8-like 2 (TIPE2). TIPE2 has been defined as a negative regulator of innate and adaptive immunity, and maintains immune homeostasis. ${ }^{9}$ TIPE2 is downregulated in patients with chronic inflammatory diseases, such as systemic lupus erythematosus, and its expression is inversely correlated with disease progression. ${ }^{10}$ The forced expression of TIPE2 in hepatocellular carcinomaderived cell lines markedly inhibits tumor cell growth ${ }^{11}$ and the proliferation of vascular smooth muscle. ${ }^{12}$ In contrast, downregulated TIPE2 is associated with a poor prognosis and promotes cell proliferation in non-small-cell lung cancer, and TIPE2-deficient $\mathrm{T}$ cells are significantly less sensitive to apoptosis. ${ }^{13,14}$ Therefore, we hypothesized that TIPE2 would have a similar effect on RA FLSs in orchestrating cell proliferation and death. More specifically, we postulated that the expression of TIPE2 would influence ZF1-induced apoptosis in AA-FLS cells.

\section{Materials and methods}

\section{Cell lines and plasmids}

AA inducement, FLS preparation, and identification techniques were conducted according to a previous publication. ${ }^{15}$ RAW 264.7 cells, normal FLSs, and AA-FLSs were grown in Dulbecco's Modified Eagle's Medium (DMEM) supplemented with $10 \%$ fetal bovine serum, penicillin, and streptomycin. To generate stable cell lines, AA-FLSs were infected with MIGR1/TIPE2 ${ }^{+/+}$recombinant lentiviral vectors, and MIGR1 lentiviral vector was used as the negative control (gift from Dr Chen Youhai, University of Pennsylvania School of Medicine). The culture medium was replaced 24 hours after infection. After an additional 48 hours, the infected cells were observed under fluorescence microscopy and infected cells constituted more than $90 \%$ of the total cell count.

\section{Antihuman DR5 single chain variable fragment $A b, Z F I$}

The anti-DR5 single chain variable fragment Ab, ZF1, reported previously in the invention patent CN 101717775A, was used in this study at $>98 \%$ purity, through a nickel ion column (Merck KGaA, Kenilworth, NJ, USA). Protein purification was performed as previously described. ${ }^{8}$

\section{Quantitative polymerase chain reaction ( $\mathrm{QPCR}$ ) and reverse transcription (RT)-PCR analysis}

Total RNA was isolated using TRIzol reagent (Invitrogen, Waltham, MA, USA) and purified with RNeasy Mini kits (Qiagen, Hilden, Germany) according to the manufacturers' instruction.

After processing with RNase-free DNase I (Invitrogen), RNA samples were reversely transcribed with oligo (dT) and SuperScript II transcriptase (Invitrogen). qPCR was performed using specific Quantitect Primers for mouse glyceraldehyde-phosphate dehydrogenase (GAPDH), TIPE1, TIPE2, and TIPE3 (Qiagen) in an Applied Biosystems 7500 system using Power SYBR Green PCR Master Mix (Applied Biosystems, Thermo Fisher Scientific, Waltham, MA, USA). Relative gene expression levels were determined using GAPDH as the control.

\section{Western blot analysis}

Aliquots of synovial cell lysates were separated with $12 \%$ sodium dodecyl sulfate-polyacrylamide gel electrophoresis, blotted onto a polyvinylidene difluoride membrane, and probed with primary antibodies (Abcam, Cambridge, UK) for 75 minutes at room temperature. The membranes were incubated with rabbit anti-actin Ab (Huaan Biotechnology, Hangzhou, People's Republic of China), anti-TIPE2 (Abcam), anti-caspase-8 (p18) (Abcam), anti-DR5 antibodies (Abcam), antinuclear factor-kappa Bp (anti-NF-кBp) 105/ p50 (phospho) (Abcam), anti-caspase-3 (pro) (Epitomics, Burlingame, CA, USA), anti-caspase-3 (active) (Epitomics), anti-caspase-9 (Epitomics), anti-Bid (Epitomics), and anti-Bax (Epitomics). The protein bands were visualized by electrochemiluminescence reactions. Immunoblot analysis was performed as previously described. ${ }^{16}$

\section{AA-FLS proliferation assay}

FLSs $\left(1 \times 10^{4}\right.$ cells/well) were added in quintuplicate to a 96-well plate (Corning Inc., Corning, NY, USA) in the presence of various concentration of ZF1 $(0.0063,0.0125,0.025$, $0.050,0.1$, and $0.2 \mathrm{mg} / \mathrm{mL}$ ); the cells were then cultured in DMEM with $10 \%$ fetal bovine serum at $37^{\circ} \mathrm{C}$ in a humidified $5 \% \mathrm{CO}_{2}$ atmosphere for 12 hours. Then, $20 \mu \mathrm{L}$ of a 3-(4,5-dimethyl-2-thiazolyl)-2,5-diphenyl-2-H-tetrazolium bromide (MTT) solution $(5 \mathrm{mg} / \mathrm{mL})$ was added to each well, and the plates were incubated for an additional 4 hours. All of the supernatant was removed before $150 \mu \mathrm{L}$ of dimethyl sulfoxide was added to each well to dissolve the formazan crystals produced by MTT. Finally, the optical density (OD) 
value was measured by a multiscanner autoreader (MR 5000; Dynatech, Chantilly, VA, USA) at $490 \mathrm{~nm}$. Inhibition ratio (\%) was calculated using the following equation:

$$
\underset{\text { Inhibition }}{\text { ratio (\%) }}=\left[\left(\mathrm{OD}_{\text {Control }}-\mathrm{OD}_{\text {Treated }}\right) \mathrm{OD}_{\text {Control }}\right] \times 100 \%
$$

\section{Detection of DR5 expression and cell apoptosis with flow cytometry}

FLSs were trypsinized gently, washed with phosphate-buffered saline (PBS, with 3\% bovine serum albumin), incubated with $1 \mu \mathrm{g}$ anti-DR5 $\mathrm{Ab}$ for 1 hour at $4^{\circ} \mathrm{C}$ washed again with PBS (with $3 \%$ bovine serum albumin), and incubated with $1 \mu \mathrm{g}$ of an anti-mouse IgG-allophycocyanin $\mathrm{Ab}$ for 30 minutes at room temperature. Finally, the cells were analyzed using a FACS scan flow cytometer (BD Biosciences, San Jose, CA, USA). For apoptosis analyses, FLSs were plated at a concentration of $5 \times 10^{5}$ cells $/ \mathrm{mL}$ in a six-well plate, incubated for 24 hours, then treated with ZF1 $(0,0.0125,0.025,0.050$, and $0.1 \mathrm{mg} / \mathrm{mL})$ for 12 hours. After treatment, the cells were harvested by $0.25 \%$ trypsin (without EDTA), washed with PBS, and suspended in binding buffer $(1 \times)$. A $100 \mu \mathrm{L}$ aliquot of the cell suspension was incubated with $5 \mu \mathrm{L}$ of annexin $\mathrm{V}-\mathrm{APC}$ and $5 \mu \mathrm{L}$ of propidium iodide for 15 minutes in the dark, then $1 \mathrm{~mL}$ of binding buffer $(1 \times)$ was added to each sample. Cells were analyzed directly by a flow cytometer as previously described. ${ }^{17}$

\section{Caspase inhibition and NF- $\kappa B$ inhibition assay}

Cells were plated at a concentration of $5 \times 10^{5}$ cells $/ \mathrm{mL}$ in a six-well plate, incubated for 24 hours. Before stimulation with $0.04 \mathrm{mg} / \mathrm{mL}$ ZF1, the TIPE2 ${ }^{+/+}-$FLS group was pretreated with caspase inhibitor Z-VAD-FMK (Sigma-Aldrich Co., St Louis, MO, USA) and the MIGR1-FLS group was pretreated with the NF- $\kappa$ B inhibitor Bay (Sigma, USA). After treatment, the cells were harvested by $0.25 \%$ trypsin (without EDTA), washed with PBS, and suspended in binding buffer $(1 \times)$ and detected cell apoptosis by a flow cytometer as previously described.

\section{Statistical analysis}

The statistical analysis was performed using SPSS 11.5 software for Windows. The data were expressed as mean \pm standard deviation, with the number of respective experiments run in triplicate. The significance of the differences in the mean values between/within multiple groups was examined by an independent-samples $t$-test and two-way analysis of variance, respectively. A value of $P<0.05$ was considered statistically significant.

\section{Results \\ TIPE2 is not expressed in AA-FLSs}

We observed an amplicon of TIPE2 in normal FLSs, but not in AA-FLSs (Figure 1A). The qPCR results were consistent with this observation (Figure 1B). To further confirm TIPE2 expression, we used Western blotting techniques in conjunction with a specific Ab against TIPE2. TIPE2 was observed in the normal FLSs in a clear band at $\sim 21 \mathrm{kDa}$, while it was absent in the AA-FLSs samples (Figure 1C and D). These results indicated that TIPE2 may play an important role in the pathology of AA.

\section{Overexpression of TIPE2 enhanced susceptibility to DR5-mediated apoptosis}

AA-FLSs were stably transfected with the recombinant TIPE2 and control MIGR1 lentivirus vectors; TIPE2 mRNA was readily detected in the TIPE $2^{+/+}$-FLS group, but not in the MIGR1-FLS group. In contrast, similar levels of TIPE1 and TIPE3 were detected in the normal FLS, TIPE2 ${ }^{+/+}$, and MIGR1-FLS groups (Figure 1E). The results demonstrated that TIPE2 overexpression did not affect the expression of other member genes within the family. This observation was also consistent with qPCR measurements (Figure 1F). Protein expression was measured using Western blotting, where TIPE2 appeared as a $21 \mathrm{kDa}$ fusion protein (Figure $1 \mathrm{G}$ and $\mathrm{H}$ ). The expression levels of the TIPE2 protein were significantly higher in the TIPE2 $2^{+/+}$-FLS group compared with the MIGR1-FLS group.

We next explored whether AA-FLSs with different TIPE2 expression levels exhibited different sensitivities to ZF1 in vitro using proliferation assays. The results showed that the cell growth inhibition rate was increased in the TIPE2 $2^{+/+}-\mathrm{FLS}$ group in a dose- and time-dependent manner (Figure 2A). We next set out to determine whether the inhibitory effect was caused by apoptosis or necrosis using flow cytometry. The rate of apoptosis for the control group was $6.62 \%-45.43 \%$, whereas the apoptosis rate for the TIPE2 ${ }^{+/+}$-FLS group was $8.56 \%-66.5 \%$ (Figure 2B). In addition, the results showed that ZF1 induced AA-FLSs' apoptosis in a dose-dependent manner, and increased TIPE2 expression enhanced the sensitivity of ZF1-mediated apoptosis in the TIPE2 ${ }^{+/+}$-FLS group in vitro.

The role of TIPE2 in DR5-induced cell death partly occurs through upregulating caspase activation while downregulating NF- $K B$ activation in TIPE2 ${ }^{+++}$-FLSs Induction of ZF1 in the TIPE2 ${ }^{+/+}-$FLS and MIGR1-FLS groups showed increased activation of caspases 8,9 , and 3 


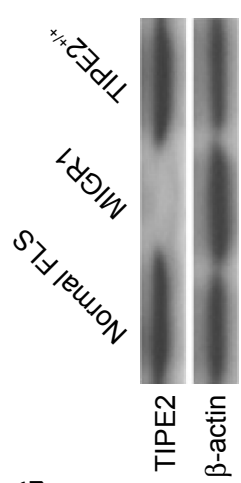

0

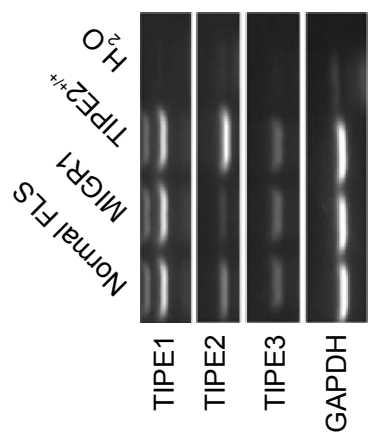

ш

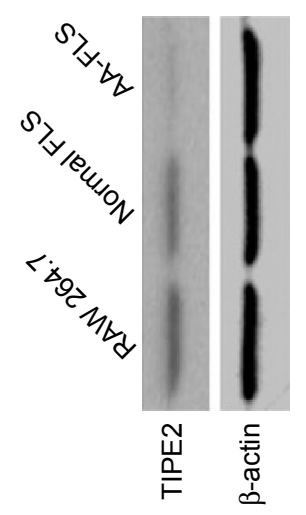

u

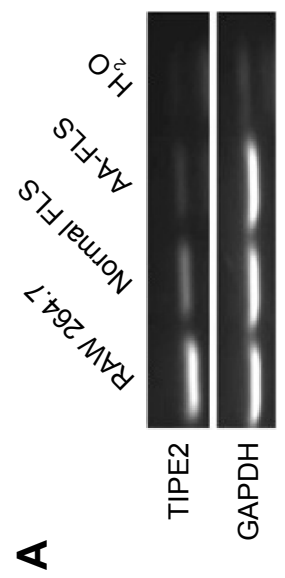

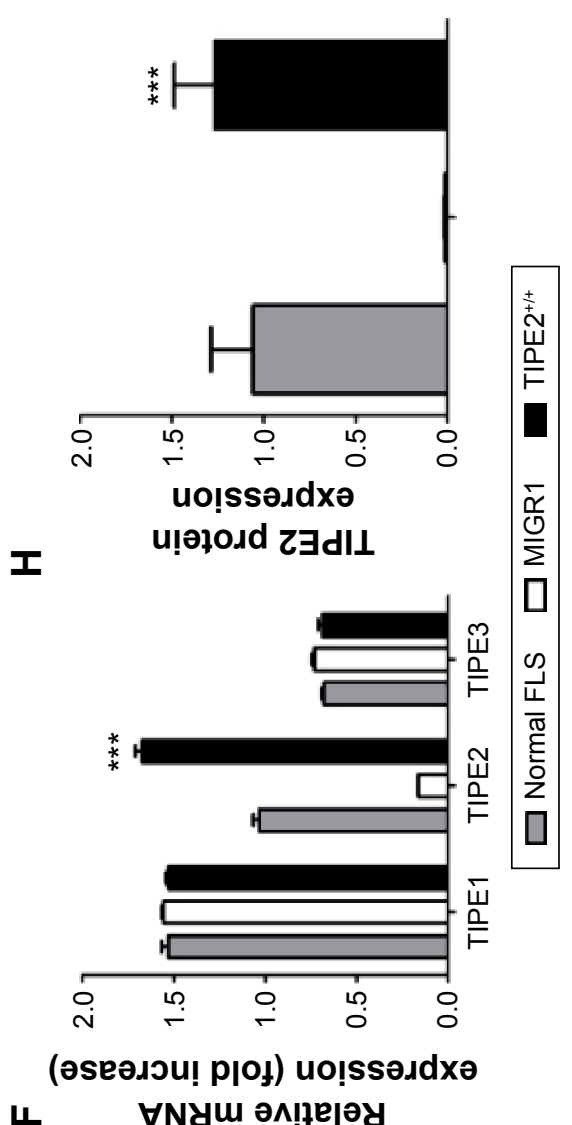

u $\quad \forall N y u$ әм!ฺе|әу

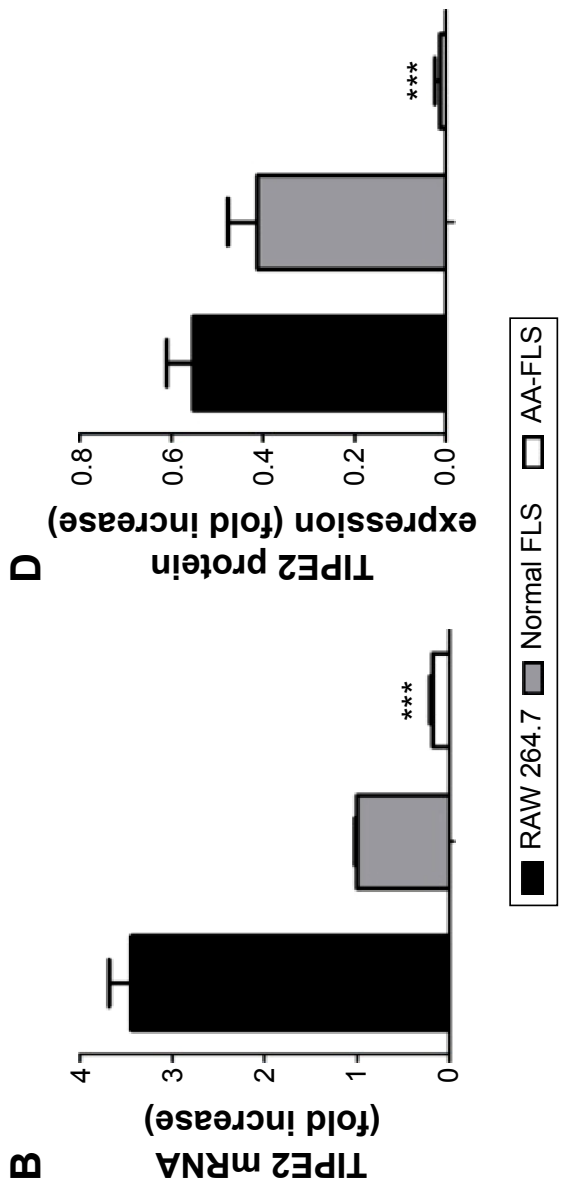

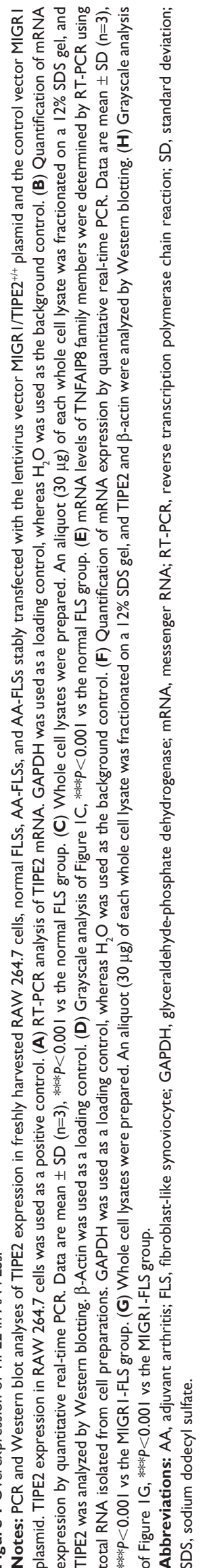




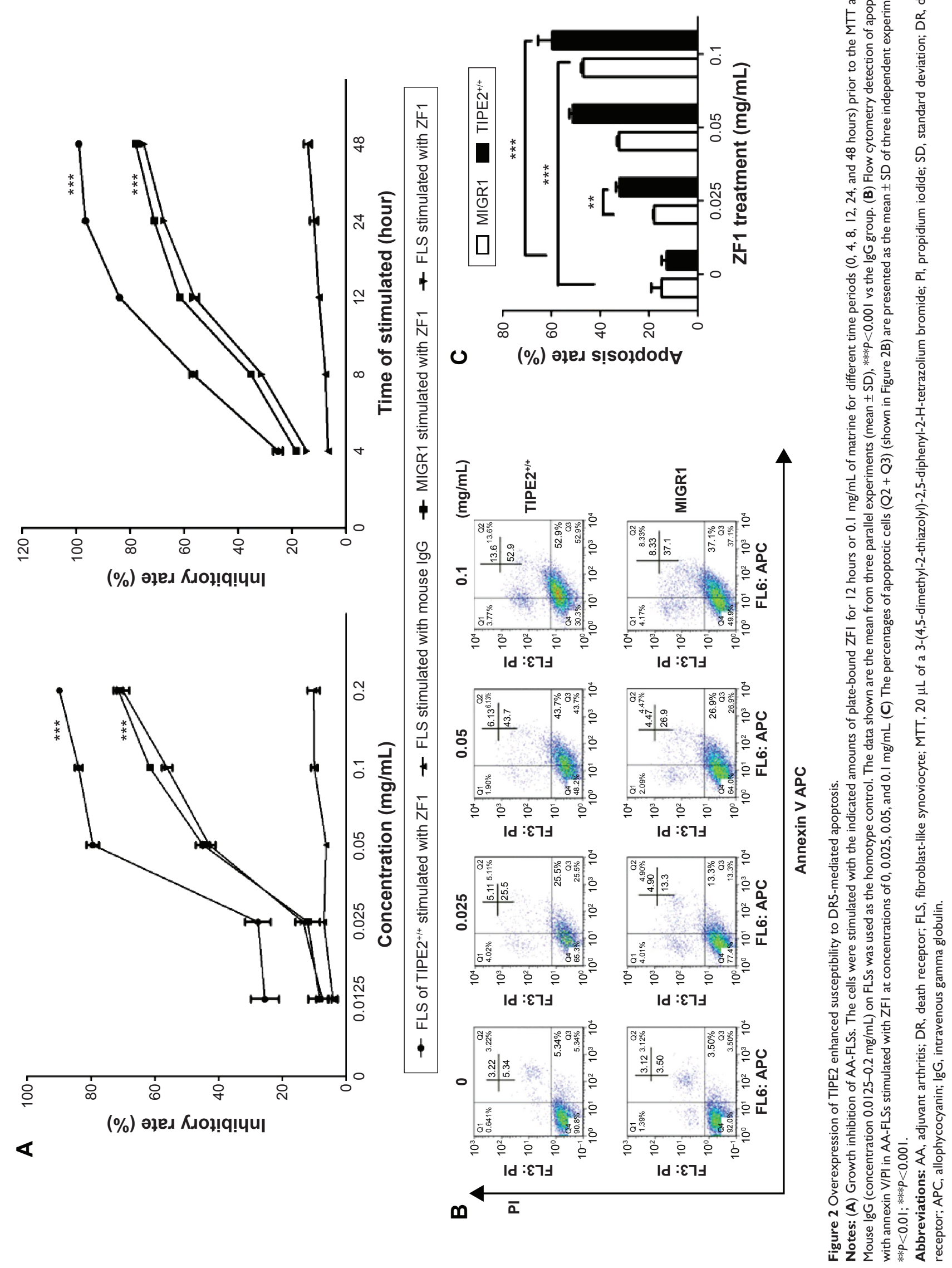


(Figure 3A). These results indicated that ZF1 is an agonistic anti-DR5 Ab, and that it induces apoptosis in a caspasedependent fashion. Thus, we showed that increased TIPE2 was able to increase the levels of apoptosis-related proteins; in this case, cleaved caspase 3 and caspase 8 were increased, while the levels of pro-caspase 3 were decreased (Figure 3A). Furthermore, to examine the mitochondrial apoptotic events associated with DR5-induced apoptosis, we analyzed the expression of its associated proteins via Western blot. The results showed that the expression of Bid was decreased,
A

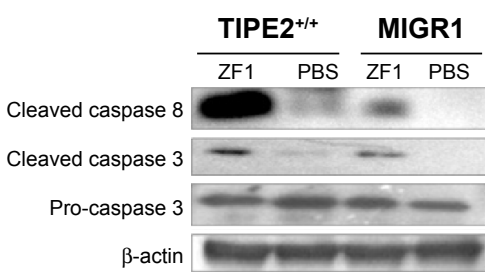

B

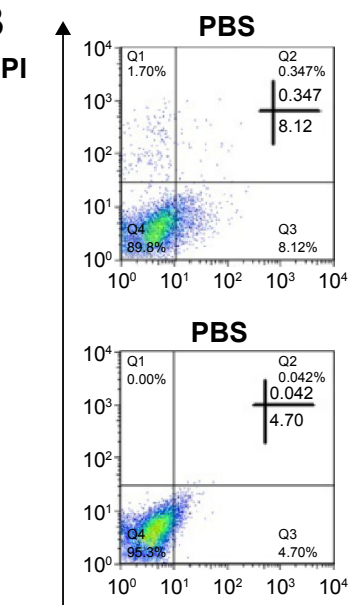

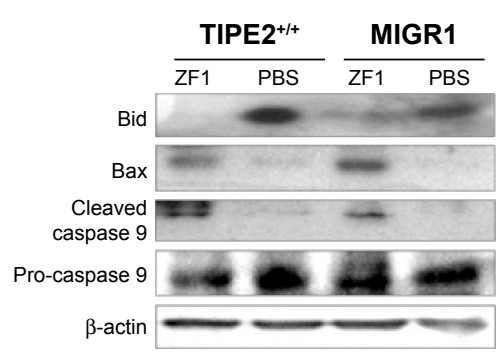

ZF1
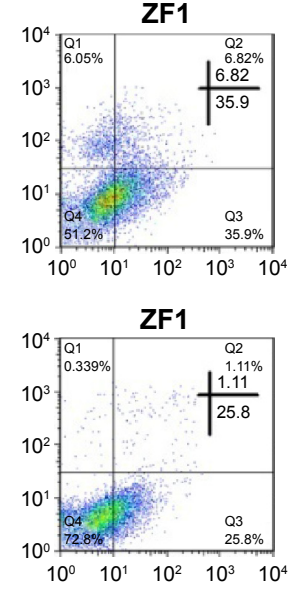

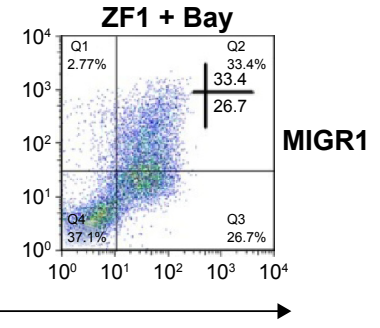

ZF1 + Z-VAD

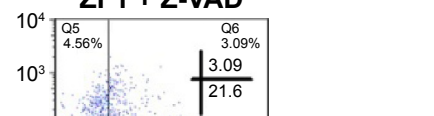

TIPE2 $^{+/+}$

Annexin V-APC
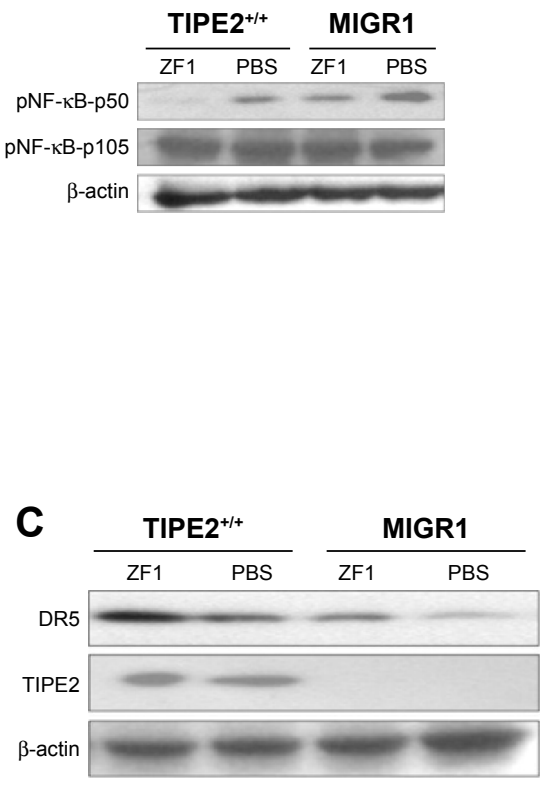
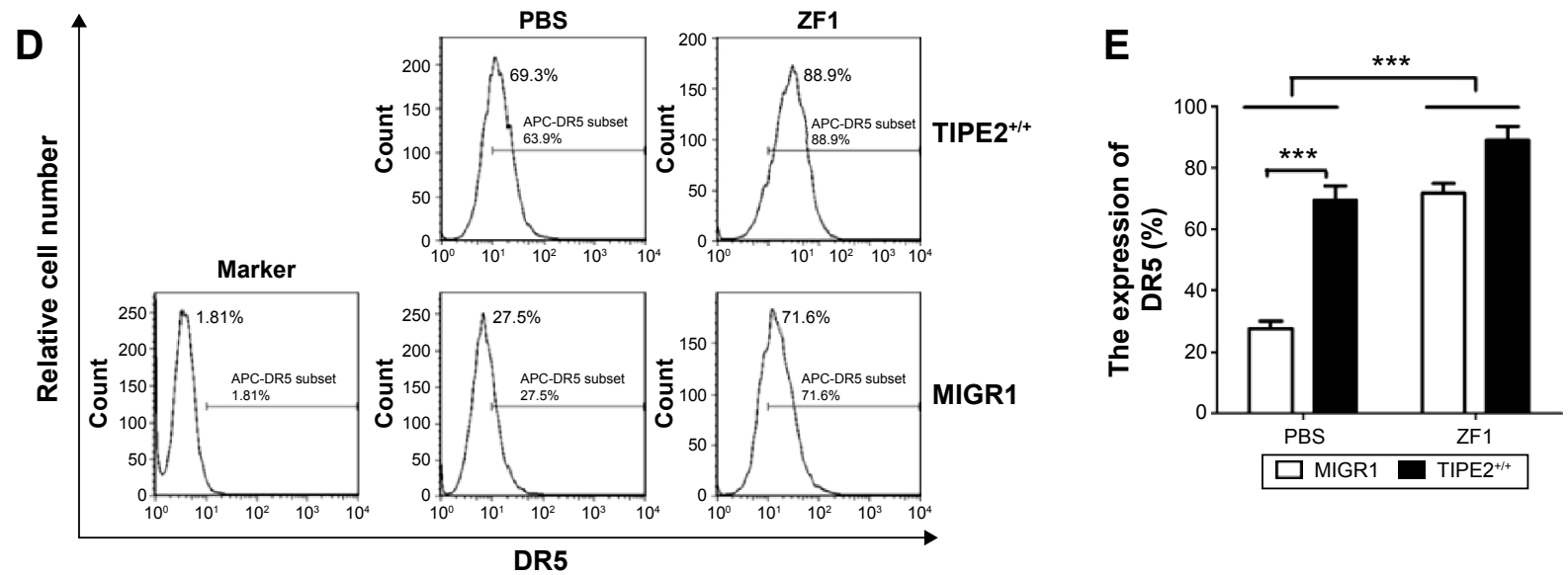

Figure 3 TIPE2 overexpression enhanced DR5 and caspase expression and downregulated the activation of NF- $\kappa B$

Notes: (A) The FLSs were stimulated with $0.1 \mathrm{mg} / \mathrm{mL}$ ZFI in ZFI groups or PBS in no ZFI groups for 6 hours. The total protein was extracted, and the expressions of apoptosisrelated and intrinsic mitochondrial pathway-related proteins and pNF-KB-p 105/p50 proteins were analyzed by Western blot assay. (B) Flow cytometry detection of apoptosis with annexin V/PI in AA-FLSs stimulated with ZFI. Before stimulation with $0.04 \mathrm{mg} / \mathrm{mL}$ ZFI, the TIPE2 $2^{++}$-FLS group was pretreated with caspase inhibitor Z-VAD-FMK and the MIGRI-FLS group was pretreated with the NF-KB inhibitor Bay. (C) The FLSs were stimulated with $0.1 \mathrm{mg} / \mathrm{mL} Z \mathrm{ZFI}$ in ZFI groups or without ZFI in PBS groups for 6 hours. The total protein was extracted and the expressions of TIPE2 and DR5 proteins were analyzed using Western blot assay. (D) TIPE2 upregulated the expression of DR5 on FLSs. Flow cytometry detection of DR5 expression with anti-DR5 and mouse lgG-APC in AA-FLSs stimulated with $0.1 \mathrm{mg} / \mathrm{mL} Z \mathrm{FI}$ or without ZFI for I2 hours was carried out. (E) TIPE2 up-regulated the expression of DR5 on FLSs. The data are presented as the mean $\pm \mathrm{SD}$ of three independent experiments, ****P<0.00I. Data are representative of three independent experiments, which showed similar results.

Abbreviations: AA, adjuvant arthritis; DR, death receptor; FLS, fibroblast-like synoviocyte; NF- $\kappa B$, nuclear factor-kappa B; PBS, phosphate-buffered saline; PI, propidium iodide; SD, standard deviation; APC, Allophycocyanin. 
whereas Bax was increased after treatment with ZF1 for 6 hours. Meanwhile, caspase 9 cleavage was significantly activated after ZF1 treatment. Such results were in line with further observation, where the mitochondrial pathway was involved in DR5-induced apoptosis (Figure 3A).

The activation of NF- $\kappa \mathrm{B}$ in TIPE2 $2^{+/+}$-FLSs was further analyzed using Western blot (Figure 3A). Compared with the control PBS group, the expression level of phosphorylated $\mathrm{NF}(\mathrm{pNF})-\kappa \mathrm{B}$ was reduced in the presence of ZF1. The level of $\mathrm{pNF}-\kappa \mathrm{B}$ was even lower in the TIPE2 ${ }^{+/+}-\mathrm{FLS}$ group treated with ZF1 than in the MIGR1 group, suggesting that TIPE2 might act as a negative regulator in NF- $\kappa \mathrm{B}$ pathways.

To further examine the molecular mechanisms involved in TIPE2-increased ZF1-mediated apoptosis, we used the caspase inhibitor Z-VAD-FMK and the NF- $\kappa B$ inhibitor Bay. The TIPE2 $2^{+/+}$-FLS group pretreated with caspase inhibitor Z-VAD-FMK showed a significantly decreased apoptosis incident. In parallel, the pretreated MIGR1-FLS group with the NF- $\kappa$ B inhibitor Bay showed an increased apoptosis rate (Figure 3B).

\section{The role of TIPE2 in DR5-induced cell death partly occurs through increasing DR5 expression in TIPE2 ${ }^{++}$-FLSs}

To determine the induction status of DR5 expression in TIPE2 ${ }^{+/+}$-FLS cells, we compared the DR5 expression in TIPE $2^{+/+}$-FLSs before and after ZF1 treatment. The protein level of TIPE2 did not change with the addition of $\mathrm{ZF} 1$, but the protein level of DR5 significantly increased (Figure 3C). DR5 expression in the TIPE2 $2^{+/+}$-FLS group was increased from $69.3 \%$ to $88.9 \%$ after ZF1 stimulation, but a much more dramatic increase was seen in the MIGR1 group, from $27.5 \%$ to $71.6 \%$ (Figure $3 \mathrm{D}$ ). These results further demonstrated that TIPE2 overexpression could enhance DR5 expression.

\section{Discussion}

RA is a chronic systemic autoimmune disorder that mainly affects joints. The pathogenesis of RA is not clear, however, it is believed to include a combination of genetic and environmental factors that cause an attack on joints from immune system. All the mechanisms result in inflammation and thickening of the joint capsule cartilage and bones. Hyperproliferation of synovial fibroblast cells is a characteristic feature of RA. Such cells have been described as "transformed-appearing synoviocytes". ${ }^{18}$

TIPE2 contains a putative death effector domain-like domain that shows significant nucleotide identity and amino acid similarity to other known DED sequences. ${ }^{9}$ Some DEDcontaining proteins are capable of regulating apoptosis. T cells with a TIPE2 knockdown showed resistance to Fas ligand-induced apoptosis. ${ }^{19}$ In parallel to the current study, the TIPE2 protein was not detected in AA-FLSs; however, it was expressed in the normal FLSs. The results reported here indicate that TIPE2 is a critical regulator of DR5-mediated apoptosis in AA-FLSs.

Our findings suggest that TIPE2 overexpression enhanced both the intrinsic and extrinsic apoptosis in AA-FLS lines, which had been induced by ZF1, and this effect was dose- and time-dependent. Ichikawa et $\mathrm{al}^{20}$ reported that RA synovial cells express high levels of DR5 and are highly susceptible to DR5-mediated apoptosis. ${ }^{21}$ Both, Li et al's ${ }^{15}$ findings and our results, suggested that AA-FLSs cells express DR5 and are sensitive to DR5-mediated apoptosis. In addition, DR5 expression was enhanced after TIPE2 overexpression in AA-FLSs, even without ZF1 treatment. Thus, we postulated that TIPE2 may regulate DR5-mediated apoptosis by altering the levels of death factors that control the DR5 apoptotic pathway. Our results suggest that caspases 8 and 3 have been significantly activated in TIPE2 ${ }^{+/+}$-FLS cells; using the caspase inhibitor Z-VAD-FMK significantly decreased apoptosis mediated by $\mathrm{ZF} 1$.

DR5 can activate the NF- $\kappa B$ pathway in addition to transducing apoptosis signals. ${ }^{22}$ Downstream, NF- $\kappa \mathrm{B}$ activation transduces an anti-apoptosis signal. ${ }^{23,24}$ Correspondingly, genetic deficiencies of the NF- $\kappa \mathrm{B}$ transcription factor increase spontaneous and TNF- $\alpha$-induced lymphocyte apoptosis. ${ }^{25} \mathrm{NF}-\kappa \mathrm{B}$ activation is significantly enhanced in TIPE2 knockdown RAW 264.7 cells. ${ }^{9}$

The apoptosis rate of the MIGR1-FLS group was increased significantly when pretreated with the NF- $\mathrm{BB}$ inhibitor Bay. These results suggest a role of TIPE2 in AAFLS apoptosis, which might be related to caspase enhancement and the inhibition of NF- $\mathrm{KB}$ activation.

Overall, we solidly demonstrated that TIPE2 protein could be detected in normal FLSs, but not in AA-FLSs. Through regulating the DR5-caspase-NF- $\kappa \mathrm{B}$ pathway, TIPE2 overexpression enhanced AA-FLS apoptosis, which was induced by the antihuman DR5 single chain variable fragment $\mathrm{Ab}$.

\section{Acknowledgments}

This work was supported by the Scientific Research Foundation for the National Nature Science Foundation of China (Grants 81072472, 81272720) and the Fujian Province Natural Science Fund (Grant 2012J01416). 


\section{Disclosure}

The authors report no conflicts of interest in this work.

\section{References}

1. Firestein GS. Evolving concepts of rheumatoid arthritis. Nature. 2003; 423:356-361

2. Bottini N, Firestein GS. Duality of fibroblast-like synoviocytes in RA: passive responders and imprinted aggressors. Nat Rev Rheumatol. 2013;9:24-33.

3. Nakano K, Boyle D, Firestein GS. Regulation of DNA methylation in rheumatoid arthritis synoviocytes. J Immunol. 2013;190:1297-1303.

4. Bartok B, Firestein GS. Fibroblast-like synoviocytes: key effector cells in rheumatoid arthritis. Immunol Rev. 2010;233:233-255.

5. Bendele A, McComb J, Gould T, et al. Animal models of arthritis: relevance to human disease. Toxicol Pathol. 1999;27:134-142.

6. Wiley S, Schooley K, Smolak P, et al. Identification and characterization of a new member of the TNF family that induces apoptosis. Immunity. 1995;3:673-682.

7. Secchiero P, Gonelli A, Carnevale E, et al. Trail promotes the survival and proliferation of primary human vascular endothelial cells by activating the Akt and ERK pathways. Circulation. 2003;107:2250-2256.

8. Yang J, Huang X, Luo F, et al. Preparation and functional studies of hydroxyethyl chitosan nanoparticles loaded with anti-human death receptor 5 single-chain antibody. Onco Targets Ther. 2014;7:779-787.

9. Sun H, Gong S, Carmody R, et al. TIPE2, a negative regulator of innate and adaptive immunity that maintains immune homeostasis. Cell. 2008; 133:415-426.

10. Li D, Song L, Fan YJ, et al. Down-regulation of TIPE2 mRNA expression in peripheral blood mononuclear cells from patients with systemic lupus erythematosus. Clin Immunol. 2009;133:422-427.

11. Cao X, Zhang L, Shi Y, et al. Human tumor necrosis factor (TNF)alpha-induced protein 8-like 2 suppresses hepatocellular carcinoma metastasis through inhibiting Rac1. Mol Cancer. 2013;12:149.

12. Zhang G, Zhang W, Lou Y, et al. TIPE2 deficiency accelerates neointima formation by downregulating smooth muscle cell differentiation. Cell Cycle. 2013;12:501-510.

13. Ruan Q, Wang P, Wang T, et al. MicroRNA-21 regulates T-cell apoptosis by directly targeting the tumor suppressor gene Tipe2. Cell Death Dis. 2014;5:e1095.
14. Li Y, Li X, Liu G, et al. Downregulated TIPE2 is associated with poor prognosis and promotes cell proliferation in non-small cell lung cancer. Biochem Biophys Res Commun. 2015;457(1):43-49.

15. Li W, Liu Z, Zhuang G, et al. Anti-DR5 mAb ameliorate adjuvant arthritis rats through inducing synovial cells apoptosis. Exp Biol Med. 2009;234:1468-1476.

16. Zhang $\mathrm{L}, \mathrm{Fu} \mathrm{X}, \mathrm{Yu}$ Y, et al. Treatment with CA-074Me, a cathepsin B inhibitor, reduces lung interstitial inflammation and fibrosis in a rat model of polymyositis. Lab Invest. 2015;95:65-77.

17. Lin Y, Chen W, Li J, et al. Overexpression of Jagged-1 combined with blockade of CD40 pathway prolongs allograft survival. Immunol Cell Biol. 2015;93(2):213-217.

18. Trabandt A, Gay R, Gay S. Oncogene activation in rheumatoid synovium. APMIS. 1992;100:861-875.

19. Gus-Brautbar Y, Johnson D, Zhang L, et al. The anti-inflammatory TIPE2 is an inhibitor of the oncogenic Ras. Mol Cell. 2012;45:610-618.

20. Ichikawa K, Liu W, Fleck M, et al. TRAIL-R2 (DR5) mediates apoptosis of synovial fibroblasts in rheumatoid arthritis. J Immunol. 2003; 171:1061-1069.

21. Seol D, Li J, Seol MH, Park SY, Talanian RV, Billiar TR. Signaling events triggered by tumor necrosis factor-related apoptosis-inducing ligand (trail): caspase- 8 is required for trail-induced apoptosis. Cancer Res. 2001;61:1138-1143.

22. Chaudhary PM, Eby M, Jasmin A, Bookwalter A, Murray J, Hood L. Death receptor 5, a new member of the TNFR family, and DR4 induce FADD-dependent apoptosis and activate the NF- $\kappa \mathrm{B}$ pathway. Immunity. 1997;7:821-830.

23. Oya M, Ohtsubo M, Takayanagi A, Tachibana M, Shimizu N, Murai M. Constitutive activation of nuclear factor-kappa $\mathrm{b}$ prevents trail-induced apoptosis in renal cancer cells. Oncogene. 2001;20:3888-3896.

24. Bernard D, Quatannens B, Vandenbunder B, Abbadie C. Rel/NF- $\kappa B$ transcription factors protect against tumor necrosis factor (TNF)related apoptosis-inducing ligand (TRAIL)-induced apoptosis by upregulating the TRAIL decoy receptor DcR1. J Biol Chem. 2001;276: 27322-27328.

25. Zheng Y, Vig M, Lyons J, Van Parijs L, Beg AA. Combined deficiency of p50 and cRel in CD4(+) T cells reveals an essential requirement for nuclear factor kappa $\mathrm{B}$ in regulating mature $\mathrm{T}$ cell survival and in vivo function. $J$ Exp Med. 2003;197:861-874.
OncoTargets and Therapy

\section{Publish your work in this journal}

OncoTargets and Therapy is an international, peer-reviewed, open access journal focusing on the pathological basis of all cancers, potential targets for therapy and treatment protocols employed to improve the management of cancer patients. The journal also focuses on the impact of management programs and new therapeutic agents and protocols on

\section{Dovepress}

patient perspectives such as quality of life, adherence and satisfaction The manuscript management system is completely online and includes a very quick and fair peer-review system, which is all easy to use. Visit http://www.dovepress.com/testimonials.php to read real quotes from published authors. 